\section{Emergence of Infections due to a Polymyxin B-Resistant KPC-2-Producing Klebsiella pneumoniae in Critically Ill Patients: What Is the Role of a Previous Colonization?}

To the Editor-Carbapenem resistance among enterobacterial species has increased worldwide and the carbapenemase production is by far the main mechanism responsible for the spread into nosocomial settings, particularly in intensive care units. $^{1}$

Early detection of colonized patients through surveillance cultures is important for the management of patients and to establish infection control practices in order to avoid carbapenemase-producing Enterobacteriaceae (CPE) dissemination. Infections due to CPE are a great concern because these organisms are highly resistant to most antimicrobial agents, leaving only a few therapeutic choices, such as polymyxins. ${ }^{2,3}$

In view of this, we conducted a retrospective survey from January to August, 2014, including patients in an adult intensive care unit of a tertiary hospital in Porto Alegre, Southern Brazil, in order to evaluate the incidence of infections by CPE recovered from some clinical sites in previously colonized patients.

Identification of enterobacterial species; determination of minimum inhibitory concentrations for ertapenem, meropenem, imipenem, and polymyxin B; search for any carbapenemase gene; and molecular typing were applied for those CPE isolates that were found in both surveillance and clinical specimens, as previously reported. ${ }^{4}$

During the period of the study, $149(24.3 \%)$ of the 613 patients were found to be colonized by CPE and in only 10 of them, a CPE was recovered in a clinical specimen, resulting in a $6.7 \%(10 / 149 ; 95 \% \mathrm{CI}, 3.7 \%-11.9 \%)$ cumulative incidence rate of infection.

Table 1 shows the 10 colonized patients with CPE who presented with a subsequent infection with the same microorganism. All these $\mathrm{CPE}$ isolates were identified as KPC-2-producing Klebsiella pneumoniae (KPC-2-KP) and all were identical by molecular typing. This finding is due to the high prevalence of a dominant clone KPC-2-KP responsible for maintaining a low endemic level in our institution, as described previously. ${ }^{5}$ For these isolates, minimum inhibitory concentrations for all carbapenems were higher than $32 \mathrm{mg} / \mathrm{L}$ whereas for polymyxin B they ranged from 0.5 to $48 \mathrm{mg} / \mathrm{L}$ (Table 1 ). It is of note that $6(60 \%)$ of these 10 patients were carrying a polymyxin-resistant KPC-2-KP. In $5(83.3 \%)$ of these 6 patients the presence of a polymyxin-resistant isolate had been previously detected in the surveillance culture.

Despite the resistance rate for polymyxin B among colonizing KPC-2-KP isolates being only $16.8 \%$ during the same period, ${ }^{3}$ our results are concerning because patients who presented with prior colonization by a polymyxin-resistant isolate ended up developing infections caused by this microorganism. Importantly, this fact shows the need to implement a rigorous protocol for polymyxin B consumption (widely used for treating infections by carbapenem-resistant Pseudomonas aeruginosa and Acinetobacter baumannii in our intensive care unit patients nowadays). Apart from that, although we have not assessed the mortality rate attributed to $\mathrm{KPC}-2-\mathrm{KP}$, this outcome is quite notable in patients infected with it (Table 1).

Colonization with potential pathogens is almost always a prerequisite for the development of nosocomial infections; however, only a minority of colonized patients eventually develops clinical infection. This relation may be influenced by many factors, including pathogen virulence, host defense mechanisms, and antimicrobial exposure. As in ours, only a

table 1. Microbiologic Characteristics and Clinical Outcome in Patients Infected With KPC-2-Klebsiella pneumoniae and Previously Colonized With This Same Pathogen

\begin{tabular}{|c|c|c|c|c|c|c|}
\hline \multirow[b]{2}{*}{ Patients } & \multirow{2}{*}{$\begin{array}{l}\text { Carbapenems } \\
\mathrm{MIC}, \mathrm{mg} / \mathrm{L}^{\mathrm{a}}\end{array}$} & \multicolumn{2}{|c|}{ Polymyxin B MIC, mg/L } & \multirow[b]{2}{*}{ Clinical site } & \multirow{2}{*}{$\begin{array}{l}\text { Use of polymyxin } \\
\text { B prior to KPC-2-KP colonization }\end{array}$} & \multirow{2}{*}{$\begin{array}{l}\text { Hospital } \\
\text { outcome }\end{array}$} \\
\hline & & Rectal screen & Clinical specimen & & & \\
\hline Patient 1 & $>32$ & 1.0 & 1.5 & Blood & Yes & Death \\
\hline Patient 2 & $>32$ & 32.0 & 16.0 & Endotracheal aspirate & Yes & Death \\
\hline Patient 3 & $>32$ & 3.0 & 2.0 & Urine & Yes & Death \\
\hline Patient 6 & $>32$ & 8.0 & 4.0 & Blood & Yes & Death \\
\hline Patient 7 & $>32$ & 8.0 & 8.0 & Endotracheal aspirate & Yes & Discharged \\
\hline Patient 8 & $>32$ & 0.5 & 48.0 & Blood & Yes & Death \\
\hline Patient 9 & $>32$ & 0.5 & 0.5 & Blood & No & Death \\
\hline Patient 10 & $>32$ & 1.5 & 1.0 & Urine & No & Discharged \\
\hline
\end{tabular}

NOTE. MIC, minimum inhibitory concentration.

${ }^{\mathrm{a} C o n s i d e r i n g} \geq 4 \mathrm{mg} / \mathrm{L}$ as resistant.

${ }^{\mathrm{b}}$ Considering $\leq 2 \mathrm{mg} / \mathrm{L}$ and $>2 \mathrm{mg} / \mathrm{L}$ as susceptible and resistant, respectively. 
few studies have addressed progression to infection among colonized patients with $\mathrm{CPE}$, using the recovery from clinical sites as a marker for infection. ${ }^{6-8}$ Interestingly, our results were similar to these studies, mainly in relation to the incidence rate of infections among colonized patients and the predominance of KPC-2-KP among the CPE isolates. On the other hand, in these studies, no data on polymyxin $\mathrm{B}$ resistance was reported.

In conclusion, despite a low incidence of infections by KPC-2-KP in previously colonized patients, a KPC-2-KP predominant clone presenting with a high polymyxin $\mathrm{B}$ resistance level has been responsible for most intensive care unit infections due to CPE isolates. Although our results should be validated by further studies, they serve as a warning to prevent the spread of polymyxin-resistant KPC-2-KP by the early detection of carriers, especially among critically ill patients.

\section{ACKNOWLEDGMENTS}

Financial support. Conselho Nacional de Desenvolvimento Científico e Tecnológico, Brazil.

Potential conflicts of interest. Both authors report no conflicts of interest relevant to this article.

\section{Leandro Reus Rodrigues Perez, $\mathrm{PhD} ;^{1,2,3}$} Cícero Gomes Dias, $\mathrm{PhD}^{3,4}$

Affiliations: 1. Programa de Pós-graduação em Ciências Farmacêuticas, Universidade Federal do Rio Grande do Sul, Porto Alegre, Brazil; 2. Laboratório de Pesquisa em Resistência Bacteriana, Centro de Pesquisa Experimental, Hospital de Clínicas de Porto Alegre, Porto Alegre, Brazil; 3. Hospital Mãe de Deus, Porto Alegre, Brazil; 4. Universidade Federal de Ciências da Saúde de Porto Alegre, Porto Alegre, Brazil.

Address correspondence to Leandro Reus Rodrigues Perez, PhD, Universidade Federal do Rio Grande do Sul, Faculdade de Farmácia, 2752 Ipiranga Avenue, 90610-000, Porto Alegre-RS, Brazil (leandro.reus@gmail.com). Presented in part: 25th European Congress of Clinical Microbiology and Infectious Diseases; Copenhagen, Denmark; April 27, 2015 (abstract no. ECCMD3386).

Infect. Control Hosp. Epidemiol. 2016;37(2):240-241

(c) 2016 by The Society for Healthcare Epidemiology of America. All rights reserved. 0899-823X/2016/3702-0025. DOI: 10.1017/ice.2015.294

\section{REFERENCES}

1. Nordmann P, Naas T, Poirel L. Global spread of carbapenemaseproducing Enterobacteriaceae. Emerg Infect Dis 2011;17: 1791-1798.

2. Carmeli Y, Akova M, Cornaglia G, et al. Controlling the spread of carbapenemase-producing gram-negatives: therapeutic approach and infection control. Clin Microbiol Infect 2010;16: 102-111.

3. Perez LR. Evaluation of polymyxin susceptibility profile among KPC-producing Klebsiella pneumoniae using Etest and MicroScan WalkAway automated system [published online September 11, 2015]. APMIS 2015;123:951-954.

4. Perez LR, Rodrigues D, Dias CG. Evaluation of phenotypic tests to detect carbapenem-resistant Enterobacteriaceae in colonized patients hospitalized in intensive care units. Braz J Infect Dis 2015;19:436-438.
5. Perez LR. Carbapenem-resistant Enterobacteriaceae: a major prevalence difference due to the high performance of carbapenemase producers when compared to the nonproducers [published online October 1, 2015]. Infect Control Hosp. Epidemiol 2015, http://dx.doi. org/10.1017/ice.2015.227. Accessed November 8, 2015.

6. Giannella M, Trecarichi EM, De Rosa FG, et al. Risk factors for carbapenem-resistant Klebsiella pneumoniae bloodstream infection among rectal carriers: a prospective observational multicenter study. Clin Microbiol Infect 2014;20:1357-1362.

7. Schechner V, Kotlovsky T, Kazma M, et al. Asymptomatic rectal carriage of $b l a_{\mathrm{KPC}}$ producing carbapenem-resistant Enterobacteriaceae: who is prone to become clinically infected? Clin Microbiol Infect 2013;19:451-456.

8. Borer A, Saidel-Odes L, Eskira S, et al. Risk factors for developing clinical infection with carbapenem-resistant Klebsiella pneumoniae in hospital patients initially only colonized with carbapenemresistant K. pneumoniae. Am J Infect Control 2012;40:421-425.

\section{CPE Clearance-A Response to Kim et al.}

To the Editor-We were interested to read the article by Kim et $\mathrm{al}^{1}$ in a recent issue of this journal. Their research confirms previous studies, including our own, regarding carriage of carbapenemase-producing Enterobacteriaceae (CPE). ${ }^{2} \mathrm{We}$ studied patients who were released from the hospital after a CPE-positive culture. We followed up with rectal swab cultures taken retrospectively and prospectively for the study or as part of clinical follow-up. In the 97 patients with follow-up cultures, mean time to CPE negativity was 387 days. At 3 months, $78 \%$ of patients (64 of 82 ) had positive cultures; $65 \%$ (38 of 58) had positive cultures at 6 months, and $39 \%$ (12 of 30) had positive cultures at 1 year. Repeated hospitalization was associated with increased duration of carriage. A small minority of our patients had a positive culture after a negative one (unpublished data), and we considered these patients to be continual carriers. Similarly, Schechner et $\mathrm{al}^{3}$ showed, in a study published in this journal, that $60 \%$ of patients (14 of 23) who had a positive follow-up screening test were screened within 3 months of the index positive culture. Thus, it is unsurprising that Kim et al found very high rates of continuing carriage during a single hospitalization.

It should be noted that both studies cited examined only KPC-type carbapenemases, which represent the major mechanism of carbapenem resistance in the United States and in our region (Israel).

Because of their findings of high rates of carriage and high rates of CRE-positive surveillance cultures after negative cultures, the authors suggest that more than 3 negative cultures are needed to consider a patient a noncarrier. However, according to their and our studies, duration of CRE carriage is expected to last throughout a given hospitalization and beyond. As such, and as is our institutional policy, given the 